\section{Ancestral asymmetry}
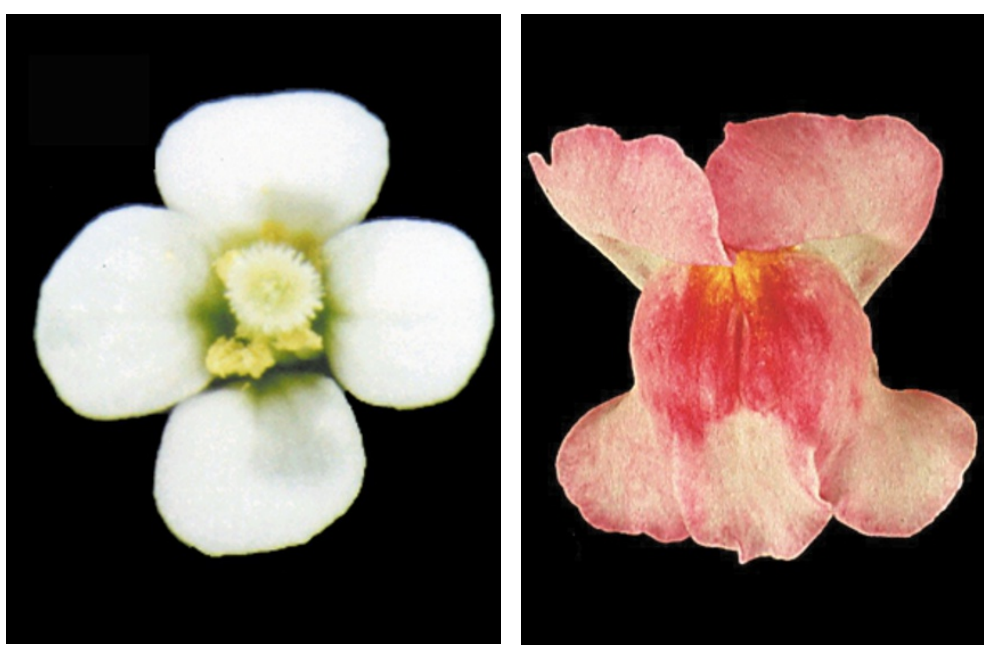

Arabidopsis thaliana (left) and Antirrhinum majus (right) flowers. A. thaliana courtesy of Pilar Cubas, INIA/CNB-CSIC, Spain. A. majus courtesy of the John Innes Centre, Norwich, UK. Reprinted from P. Cubas et al. Current Biology 11, 1050-1052 () (2001) with permission from Elsevier Science.
Unlike humans, who are said to be attracted by symmetrical (facial) features, pollinating animals are partial to the asymmetrical shape of the flowers that they help fertilize. Asymmetrical flowers have arisen many times in the evolution of flowering plants, but it is unknown whether the same molecular mechanisms have been used over and over again, or whether new strategies have been deployed each time. Pilar Cubas and colleagues show that the asymmetrical expression of a gene that controls floral asymmetry is found even in the meristems (undifferentiated cells) that develop into symmetrical flowers. This indicates that an ancient, radially symmetrical ancestor might have had an incipient asymmetry, from which asymmetrical flowers have arisen repeatedly by the same molecular route.

The flowers of the mustard weed Arabidopsis thaliana are symmetri- cal, unlike those of its distant relation, the snapdragon Antirrhinum majus, which have dorsoventral asymmetry (see photo). In the snapdragon, floral asymmetry is conferred by the asymmetric expression of a TCP-transcription factor encoded by the gene CYCLOIDEA (CYC). The gene is dorsally expressed from the early stages of development in the floral meristems, where it affects the growth rate of the meristem and so, later on, the asymmetry, size and shape of the reproductive organs. Cubas et al. found that the expression of the A. thaliana orthologue of $C Y C, T C P 1$, was also expressed in the dorsal part of flower meristems, but only very early in development. This limited expression might explain why $A$. thaliana has symmetrical flowers. Molecular and morphological phylogenies agree that the common ancestor of the two species lacked dorsoventral

\title{
Neurodegeneration studies forge ahead
}

\author{
Iron is an essential element, and its ability \\ to readily lose and accept electrons makes it \\ a vital part of the enzymes that catalyse \\ redox reactions. But this property also \\ makes iron a hazard to cells as it catalyses \\ the formation of free radicals, which \\ damage cellular structures and DNA. To \\ prevent this, some organisms have evolved \\ ways to sequester and transport iron \\ around the body. In humans, increased iron \\ deposition in the brain's basal ganglia \\ occurs with ageing and markedly in several \\ neurodegenerative diseases, such as \\ Huntington and Alzheimer. Two groups \\ now report genetic defects in two human \\ neurodegenerative disorders characterized \\ by such deposition, strongly indicating that \\ iron accumulation has a key role in the \\ aetiology of neurodegeneration. \\ Bing Zhou and colleagues mapped \\ Hallervorden-Spatz syndrome (HSS) - an \\ early-onset, recessive, neurodegenerative \\ disorder - to a region on chromosome 20 \\ in an Amish pedigree. By screening for \\ mutations in brain-expressed, candidate \\ genes in this interval, they found a 7-bp
}

deletion in a gene called PANK2 in this pedigree and in other HSS patients. The ubiquitously expressed $P A N K 2$ encodes pantothenate kinase, an enzyme in the coenzyme A (CoA) biosynthetic pathway. But how does altered CoA synthesis lead to specific neurological defects? PANK2 mutations most likely cause neurodegeneration secondarily to their effect of blocking the CoA pathway by causing secondary metabolites to accumulate. Indeed, such a metabolite, cysteine, accumulates in the basal ganglia of HSS patients to potentially lethal effect because cysteine not only is cytotoxic but also produces free radicals in the presence of iron. Such a combination could damage neuronal cells that are already stressed by impaired membrane biosynthesis caused by depleted CoA levels, so leading to cell death and neurodegeneration.

Unlike the secondary neurodegenerative effects of PANK2 mutations, in a novel, dominantly inherited, late-onset disorder studied by John Burn's group neuroferritinopathy - neurodegeneration is probably caused by the altered storage of iron. This group identified a mutation in $F T L$, which encodes ferritin light chain - a subunit of ferritin, the molecule that stores and sequesters free iron - in an English pedigree. FTL was considered a candidate gene because of its map position and the suspected involvement of iron in neurodegeneration. The same mutation was found in five other neurodegenerative disease cases, who share a founder haplotype with the original pedigree. Because of the disorder's rarity, Curtis et al. believe that the FTL mutation acts as a dominant negative by encoding a structurally altered subunit that is incorporated into ferritin, disrupting its function, perhaps by preventing the normal transfer of iron into and out of this molecule. Studies in mice will no doubt follow these exciting findings, providing greater insight into how iron accumulation contributes to the cause and progression of neurodegenerative disease. 
asymmetry; the authors propose that this ancestor had an asymmetrical 'pre-pattern' - evident from early CYC expression - which could be readily elaborated to evolve asymmetry.

There are other examples of morphological traits that have arisen many times in evolution, such as the emergence of visual organs, although in most cases their mechanisms are not known. This study provides a possible explanation for how asymmetrical flower shape evolved repeatedly using essentially the same mechanism; however, a more definitive picture will only emerge from analysing a wider range of plant species.

Tanita Casci

(2) References and links ORIGINAL RESEARCH PAPER Cubas, P. et al. Ancient asymmetries in the evolution of flowers. Curr. Biol. 11, 1050-1052 (2001) WEB SITE Enrico Coen's lab

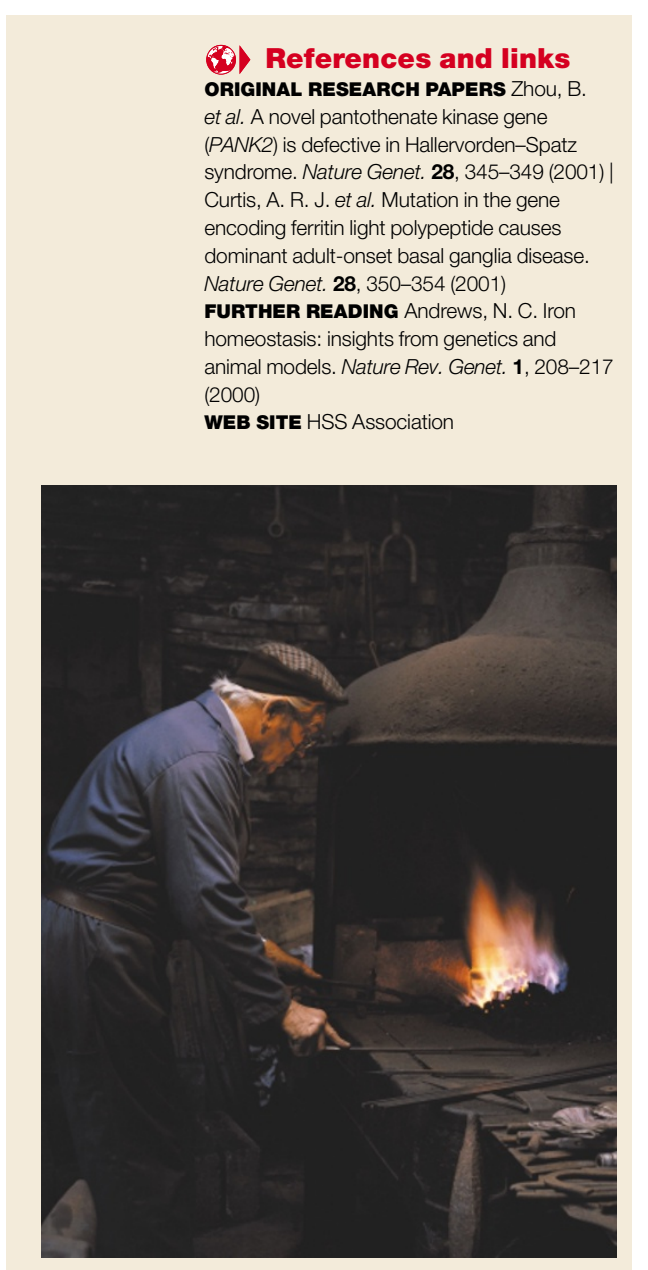

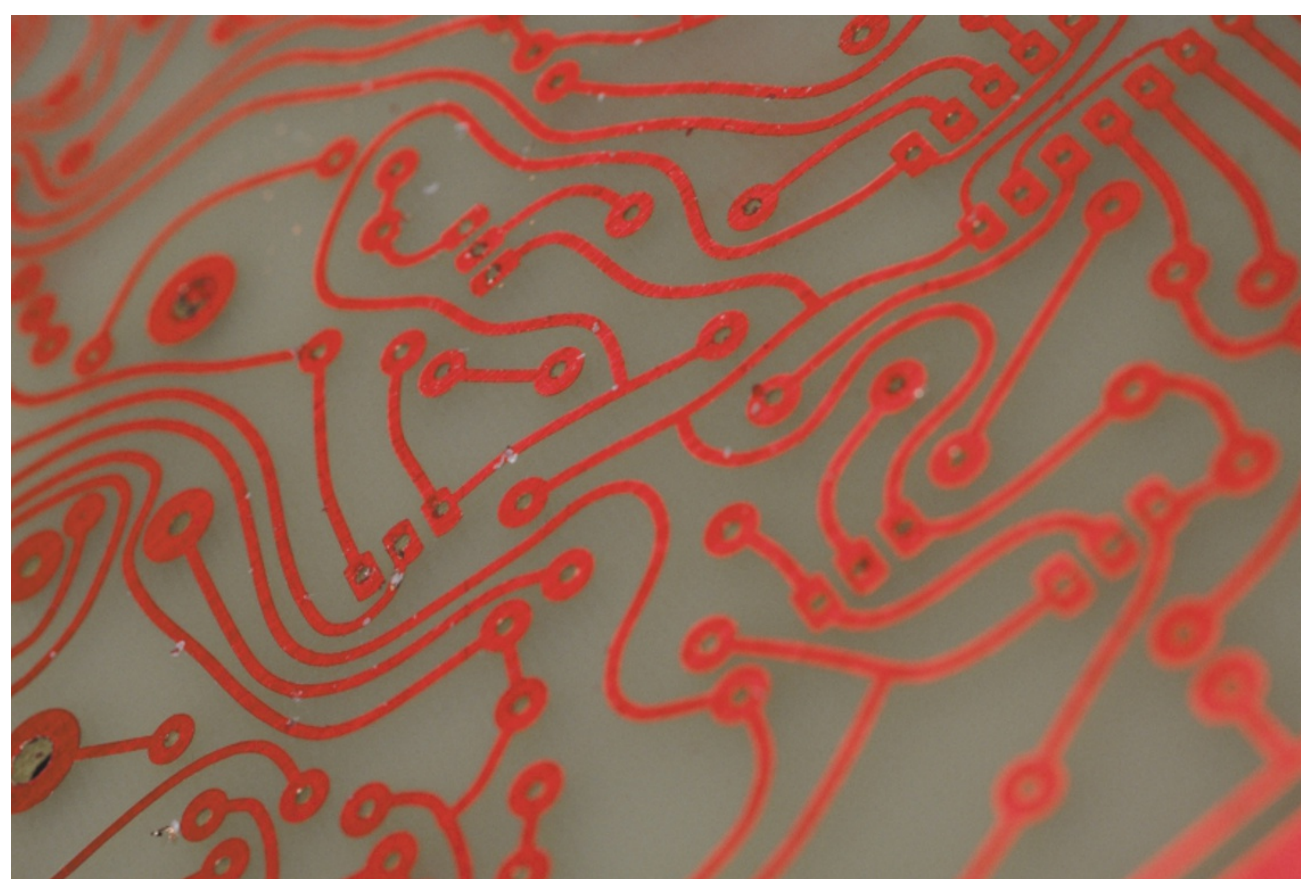

\section{FUNCTIONAL GENOMICS}

\section{The logic of transcription}

Microarray technology provides a powerful vantage point from which to study the regulation of gene transcription. As well as surveying the transcriptional profile of a cell, microarrays can also be used to find all the in vivo genomic targets for any transcription factor (as featured in February's Highlights). This was the goal of two recent studies of gene transcription in budding yeast, which show how we might advance to a comprehensive description of the circuitry and logic that underlies gene regulation.

Frédéric Devaux, Philippe Marc and colleagues focused on the transcription factor Pdr1 - a protein involved in pleiotropic drug resistance in yeast. First, they fused the DNA-binding domain of Pdr1 to the transcription-activation domain of Gal4. Then, they used microarrays to compare the genes regulated by the Pdr1-Gal4 fusion protein with the genes regulated by a constitutively expressed allele of $P d r 1$. The transcriptional profiles were almost identical, which supports the idea that the DNA-binding domain of Pdr1 is the sole determinant of DNA-binding specificity. In total, 23 genes were upregulated by Pdr1, out of 218 genes that contain a sequence that matches the consensus binding site for Pdr1. It remains to be determined how the Pdr1 DNA-binding domain discriminates between these potential binding sites. However, this approach might be a useful way to find sites bound by any DNAbinding domain, although the authors point out that this will only be appropriate for proteins in which the DNA-binding domain is autonomous and clearly defined.

A more direct approach was used by Jason Lieb et al. to identify the genomic DNA sequences bound by the transcription factor Rap1. After inducing protein-DNA crosslinks in vivo, DNA bound to Rap1 was immunoprecipitated using a Rap1 antibody. This DNA was then hybridized to microarrays of yeast genomic DNA, to identify sequences that were enriched in the immunoprecipitated fraction. Rap1-binding sites were identified at a resolution of around $2 \mathrm{~kb}$ in the promoters of $362 \mathrm{ORFs}$, including 122 ribosomal protein genes and another 185 previously unknown targets of Rap1.

A comparison of the genomic sequences bound by Rap1 allowed Lieb et al. to define a consensus sequence for Rap1 binding, which agreed with the previously determined binding site. So, this approach could be used to identify the sequence bound by a DNA-binding protein, the target site of which is unknown. However, as might be expected, the sequence of the binding site is not the only determinant of binding to Rap1. As in the Pdr1 study, Rap1 binds only a small subset of the consensus binding sequences in the genome, strongly preferring consensus sequences that are in promoters rather than those found in ORFs or in non-promoter intergenic regions. The authors suggest that the reason for this lies in the broader context of the consensus sequence, which might affect interactions with other transcription factors or chromatin structure.

Having identified all the genes bound by a transcription factor, it is still necessary to find out how many of those genes are regulated by the factor - a question that Lieb et al. address using transcriptional profiling. Once this type of information is available for many transcription factors, we will have a spectacular view of genetic regulatory circuitry.

Mark Patterson

\section{(9) References and links}

ORIGINAL RESEARCH PAPERS Devaux, F. et al. An artificia transcription activator mimics the genome-wide properties of the yeast Pdr1 transcription factor. EMBO Rep. 2, 493-498 (2001) | Lieb, J. D. et al. Promoter-specific binding of Rap1 revealed by genome-wide maps of protein-DNA association. Nature Genet. 28, 327-334 (2001) WEB SITES Claude Jacq's lab | Pat Brown's lab 